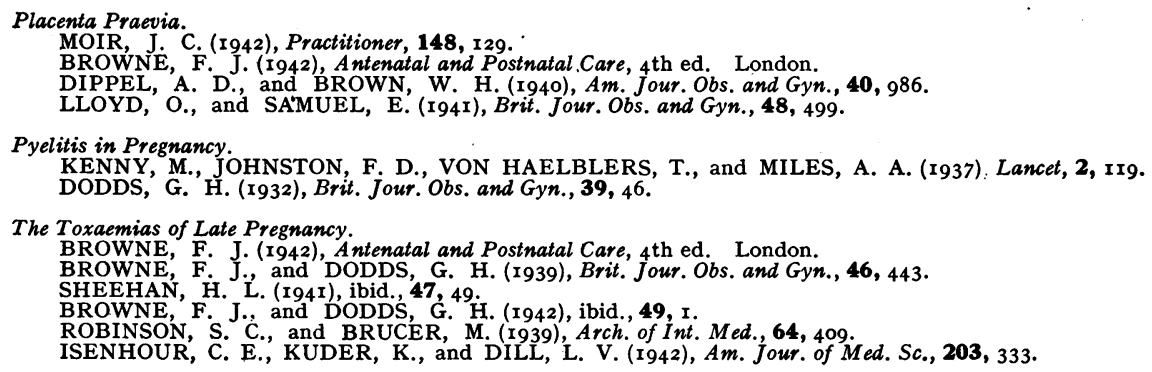

\title{
Practicalities
}

(Vol. I. No. 6)

\section{THE ELECTROCARDIOGRAPH IN DIAGNOSIS OF CARDIO-VASCULAR DISEASES}

\author{
By F. CROXON DELLER, M.D., D.A.
}

Before discussing some of the abnormal electrocardiograms which will be found in disease it would be well to consider the principles underlying the electrocardiograph.

If a strip of muscle is isolated and connected at each end to the poles of a galvanometer, the application of a single electrical stimulus to one end of that strip will cause a contraction at the point stimulated. This area immediately becomes electrically negative in relation to the unstimulated end, and an electrical current flows from positive to negative, causing a deflection, say, to the left, of the galvanometer needle. The contraction wave then passes along the muscle strip so that when it arrives at the centre of the muscle strip the negative charge is in the middle, whilst the two ends hold a positive charge; when this occurs no current flows through the galvanometer. The needle of the galvanometer will therefore come to rest again in the central position. The contraction wave continues, and on arrival at the end opposite to that originally stimulated, this becomes negatively charged and the direction of flow of the electrical current is reversed, the galvanometer swinging over to the right. These deflections can be recorded by means of the reflection of a beam of light from a mirror attached to the needle of the galvanometer; it can be arranged so that a flow of current in one direction will cause a deflection of the beam of light, say, in an upward direction, whereas a flow of current in the opposite direction will cause a deflection in a downward direction. Once, therefore, standardisation of the flow of current has been obtained, it will be possible to collate the various deflections produced in a strip of muscle, and to make deductions from these changes of any abnormalities that may be present in the muscular contraction.

Normally, in clinical electrocardiography three leads are used, by placing the electrodes upon the left wrist, the right wrist and the left leg just above the ankle. From the electrical point of view these leads are at an infinite distance from the heart muscle and measure the changes in electrical potential which are occurring due to the cardiac contractions. These leads record the changes produced firstly across the base of the heart, secondly along the right border, and thirdly along the left border of the heart, and are known as Lead I, taken from left arm to right arm, Lead II, from right arm to left leg, and Lead III, from left leg to left arm. Further research into electrocardiographic changes showed that it was often possible to detect slight changes in electrical potential not recorded by these classical leads, by moving one or two of the leads and placing them much nearer to the heart. This is known as Lead IV, or the chest lead, and its significance will be discussed in relation to coronary diseases.

The normal standardisation employed is that I millivolt change of potential will cause a deflection either upward or downward from the isoelectric line of I centimetre in height; the isoelectric line being that line produced upon the record when no changes of electrical potential are occurring. 
The graph upon which these changes are recorded is usually made up of a number of large squares, each of which represents, in length, one-fifth of a second, and in height, half a millivolt change of potential. These large squares are further subdivided into five smaller squares, each of which therefore represents in time 0.04 seconds, and in height 0.1 millivolt change of potential. But with certain types of electrocardiographs, for example the Cossor-Robertson electrocardiograph, there is a time marker measuring $0 \cdot$ I second only.

It has been found that the normal action of the heart produces certain changes of potential which can be recorded, and so form the normal electrocardiogram (Fig. I). For ease of description the various waves have been named the $P, Q, R, S$ and $T$ waves. It will be remembered that normally the impulse which causes cardiac contraction starts in the sino-auricular node and passes over the auricular muscle. Such impulses are then collected together at the auriculoventricular node and pass down the auriculo-ventricular bundle and through its main branches to the terminal Purkinje fibres, which are situated within the ventricular muscle. It has been

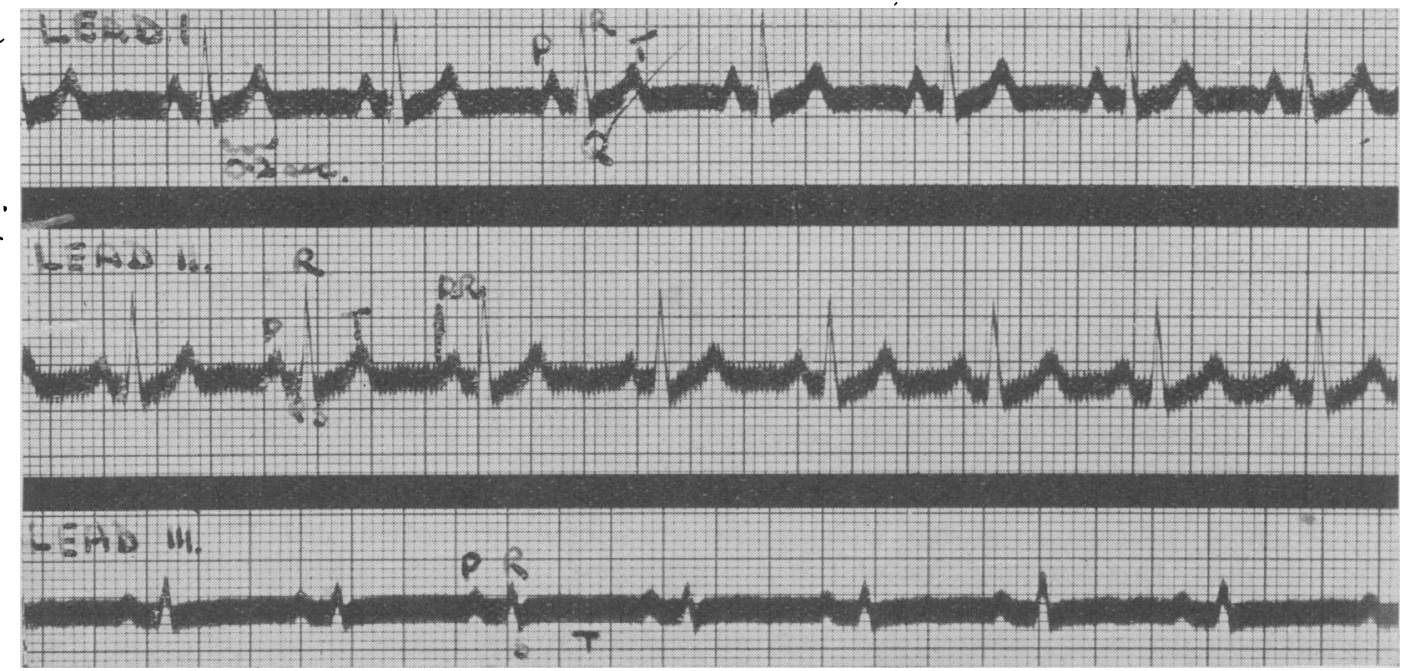

FIG. I.-Normal E.C.G. Ventricular rate 84. No axis deviation. The waves are marked in each lead. $T_{8}$ is small and inverted.

found that $\mathrm{P}$ represents auricular contraction, and is a small wave which arises about $0 \cdot 2$ centimetres above the isoelectric line and lasts for about $0 \cdot I$ of a second, or less. This is separated from the main ventricular complex, which has been named QRST, by an interval loosely known as the PR interval. This represents the time taken for the impulse to pass from the sinoauricular node to the auriculo-ventricular node, and is normally of between $0 \cdot 13$ to $0 \cdot 16$ second in length, but it is not considered abnormal until it is greater than 0.2 second. The ventricular contraction is represented by the QRST complex on the record. The $Q$ wave is often of very small amplitude, and is in a downward direction, but it may in many cases be absent. The wave which is so characteristic of the ventricular contraction is the large, upward spike termed $\mathbf{R}$, which is followed by an equally quick downward deflection to the isoelectric line, which may, however, dip slightly under that line, and is then termed the $\mathrm{S}$ wave. The QRS complex is usually 0.08 second in length, and is considered abnormal if it is spread over more than $0 \cdot I$ second. This complex is followed by an isoelectric period, after which a large $T$ wave occurs, which is usually upright. The $T$ wave may arise half a centimetre in height, and it lasts for about 0.27 second. The total time taken for the electrical changes to occur, from the beginning of the $Q$ or $R$ wave to the end of $T$ is 0.43 second or less. The $T$ wave is followed by another isoelectric period, known as the TP interval which represents the diastole of the heart. These waves, then, constitute the normal electrocardiogram, and variations from this normal graph must be interpreted with accuracy.

Upon scrutiny of the normal electrocardiogram made up of Leads I, II and III, the following points must be remembered. Firstly, it is most important that the ventricular rate be known. 
The usual electrocardiographic tracing represents 5 seconds in time, and therefore, by counting the ventricular complexes in each of the three leads, taking their average and multiplying by twelve, the ventricular rate per minute will be obtained. The next thing to be looked for is whether or not there is any axis deviation. The theory of axis deviation will not be discussed here, but from the practical point of view, if the $R$ wave is largest in Lead $I$, with a corresponding large S wave in Lead III-that is, the main ventricular spikes are in opposite directions in Leads I and III-this may be interpreted as left axis deviation, and signifies that the musculature of the left ventricle has undergone hypertrophy. This is commonly seen in diastolic hypertension. On the other hand, the main ventricular spike may be an $\mathrm{S}$ wave in Lead I and an $\mathrm{R}$ wave in Lead III-that is, these spikes are pointing towards one another-this signifies right axis deviation, and is due to hypertrophy of the right ventricle, as seen in those conditions in which there has been a heightened pressure within the pulmonary circuit, such as in old-standing cases of mitral stenosis, or following left ventricular failure.

The next thing to examine is the form of the PQRST waves, and it is usual to do this first in Lead II, as this lead generally gives the maximal deflections. The main features of these complexes have already been discussed, but, to reiterate, $\mathrm{P}$ should be upright, followed by a PR interval of under 0.2 second, $Q R S$ should be under 0.08 second, and the $T$ wave should be upright. The QT interval should be $0 \cdot 43$ second or less. TP represents diastole, and therefore will vary in duration with the ventricular rate. Any curve not complying with these findings should be considered abnormal. The complexes may then be examined in Leads I and III, but it must be remembered that the finding of an inverted $T$ in Lead III has no pathological significance.

We will now consider briefly the changes which are produced by the irregularities of the heart:-

(I) Sinus arrhythmia: In this condition the only alteration that is found is a changing TP interval, due to the increased output of stimuli from the sino-auricular node during inspiration, and a decreased output during expiration. Otherwise both auricular and ventricular complexes are normal.

(2) Extrasystoles: The extrasystole is due to the interpolation of another stimulus, causing contraction of the cardiac muscle, which arises from some focus other than the sino-auricular node. It may, therefore, be one of the following three varieties-auricular, ventricular, or A.V. nodal, depending upon the site of the more excitable area of the myocardium. The auricular extrasystole is shown on the electrocardiogram by the appearance at some time before the normal auricular complex should arise, of an abnormal $\mathrm{P}$ wave. It is usually of an inverted type, and is followed by a normal QRST complex and then a compensatory lengthening of the TP interval. The ventricular extrasystole is much the most common type, and it is shown by the interpolation of an abnormal QRST complex at some time before the normal auricular complex should appear. The QRST is of abnormal type, with much spreading of the QRS complex. It may be seen that in a case with multiple extrasystoles, many of these abnormal complexes differ in form, showing that the stimuli are arising from various parts of the ventricular myocardium. The $T$ wave which follows is often normal in type, but may be larger or smaller than normal. The TP interval is again longer than usual. The auriculo-ventricular nodal type of extrasystole is the most rare, and is due to an impulse starting from a focus somewhere within the region of the A.V. node. It may be difficult to differentiate a $\mathbf{P}$ wave at all, since it is lost in the ventricular complex which occurs simultaneously with the auricular contraction; the QRS complex therefore becomes changed, with some spreading; or the $\mathrm{P}$ wave may'occur just before $\mathrm{R}$ or just after the $\mathrm{S}$ wave, and be inverted. The $\mathrm{T}$ wave is usually normal in type, and there is again an increased TP interval following this premature contraction.

(3) Heart block: In its most severe form, that is, when the auricles and ventricles beat at completely independent rates, and therefore known as idioventricular rhythm, heart block shows itself upon the electrocardiogram in the following way: The ventricular complexes are of normal type, but occur at very slow intervals, the rate being about 30 beats to the minute, that is, two or three complexes occur on each lead record. The auricular complexes producing a normal $\mathrm{P}$ wave also occur at regular intervals, but at a much faster rate, usually about 72 beats to the minute (Fig. 2). Therefore one sees a regular sequence of $P$ waves which are not followed by QRST waves. The ventricular complexes are also normal, and occur at regular intervals, but they bear only an occasional coincidental relationship to the $P$ wave. In the lesser degrees of heart block the only sign may be an increasing length of the PR interval in 
successive beats; and after a certain number of beats the conduction down the auriculo-ventricular bundle is so slowed that the auricular contraction is not followed by a ventricular complex at all, producing an occasional dropped beat. After this, the process is again repeated. In the more severe grades of heart block the auriculo-ventricular bundle may be incapable of

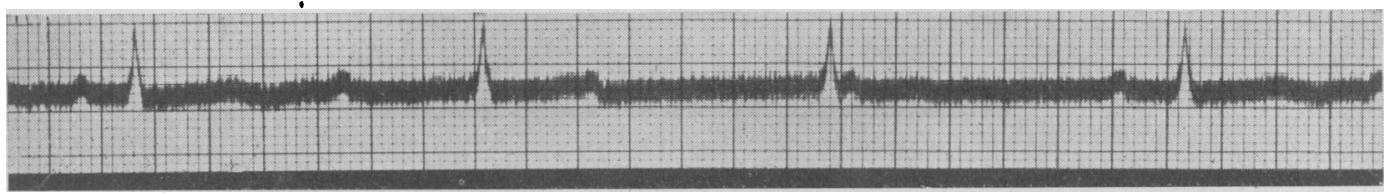

FIG. 2.-Complete Heart Block. Auricular waves occur at regular intervals, rate 72. Ventricular complexes occur at regular intervals, rate 48 . RT intervals just over $0.44 \mathrm{sec}$. Note complete dissociation of the auricular and ventricular complexes.

transmitting every beat generated at the sino-auricular node; therefore it only transmits every second, third or fourth stimulus from the sino-auricular node. In this case one will see two or three auricular complexes occurring before each ventricular complex, in a regular sequence; this is known as a $2: \mathrm{I}$ or $3: \mathrm{I}$ heart block.

(4) Paroxysmal tachycardia : In this condition a new focus of stimulus formation arises on some part of the myocardium. There may be three varieties of paroxysmal tachycardia, auricular, ventricular or A.V. nodal. The clue to diagnosis is given by the ventricular rate, which is above 160 beats per minute. In the auricular variety, the ventricular complexes are normal in shape, being separated by an abnormal $\mathbf{P}$ wave which is usually of inverted type, or it may be merely abnormal for the patient, i.e. an electrocardiographic tracing taken between the attacks will show a different form of $\mathrm{P}$ wave. In the $A . V$. nodal type, the $\mathrm{P}$ wave is usually inverted and follows the QRS complex, or the $P$ wave will not be made out at all. A ventricular tachycardia may, on rare occasions, be present. In this form no $\mathrm{P}$ wave is found, and the QRS complexes are wide, and notched, followed by a large $T$ in the direction opposite to the main deflection of the QRS complex.

It must be emphasised, however, that paroxysmal tachycardia, although usually showing itself clinically by a tachycardia between $\mathrm{I} 60$ and 210 beats per minute, may be present with a rate as low as Ioo. The electrocardiogram will make the diagnosis in any uncertain case.

(5) Auricular fibrillation : In auricular fibrillation there is no longer an output of stimulus formation from the sino-auricular node; it is replaced by a circus movement which passes in an irregular manner around the auricles. Therefore, the ventricular complexes which occur are of normal shape, but occur at irregularly spaced intervals; and in untreated cases, at a

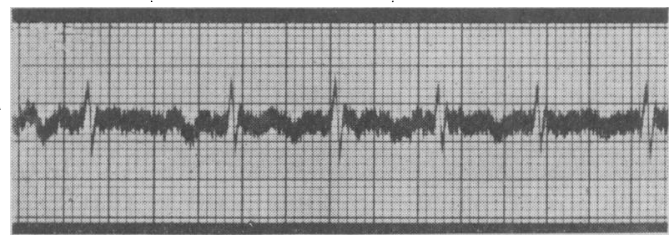

FIg. 3.-Auricular fibrillation. Ventricular rhythm irregular, rate about 84. Auricular wave replaced by small irregular fibrillary tracings, the $f$ waves.

much higher rate than normal (Fig. 3). Between the complexes are found small, irregular contractions which do not deviate greatly from the isoelectric line. These are known as $f$ waves. No $P$ wave is present.

(6) Auricular flutter: In this condition the stimulus to ventricular contraction again arises from a stimulus which passes in a circular manner around the auricle but over the same route each time. The ventricular complexes which occur are therefore of normal type, and usually occur at regular intervals, but the auricular contractions produce $P$ waves which will be found to occur three, four or five times as often as the ventricular contractions, because the auricles are contracting at a much higher rate than the ventricles. This is spoken of as a 2 , 3 or 4: I block in flutter. In some cases, however, the block is not constant, causing a changing 
degree of block to occur and an irregularly-spaced ventricular response (Fig. 4). Furthermore, in some cases of flutter, exercise will cause a sudden doubling of the ventricular rate, and therefore halving of the block which has been present in a previous record.

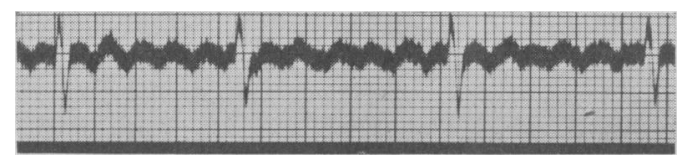

FIG. 4--Auricular flutter. Ventricular complexes of normal contour, rate 84 and regular. Auricular waves of approximately normal contour, regular, rate 420. 'This tracing shows a block which varies between $4:$ I and $5:$ I.

(7) Pulsus alternans : In this condition the beats which are felt at the wrist come through as alternate strong and weak pulsations. The most common cause of this is a rapid heart action, as found in such conditions as paroxysmal tachycardia or auricular flutter; therefore the electrocardiographic tracing will show these conditions only. There is, however, a condition known as an electrical alternans, in which each alternate contraction of the ventricle is shown on the electrocardiogram to be of a lessened amplitude than the contraction preceding it. It must not be confused with the clinical condition of pulsus alternans. Electrical alternans usually implies gross myocardial change.

(8) Auriculo-ventricular nodal rhythm is a rare condition in which the stimulus to contraction arises from or about the auriculo-ventricular node. The rate is usually slower than normal-about 60-and the auricular contraction is shown by an inverted $P$ wave which occurs either just before or just after the ventricular complex. It may, however, be incorporated in the ventricular complex, which therefore becomes abnormal in this case.

(9) Ventricular fibrillation is an extremely serious condition, but it may be found in those cases in which an electrocardiographic tracing is taken during a Stokes-Adam's attack since it is one of the causes of these attacks. The ventricular complexes occur at a rapid rate and are completely abnormal, showing marked spread, and no prior $\mathrm{P}$ wave can be made out.

Before considering the electrocardiographic changes which may be found in disease of the coronary arteries, mention must be made of the chest lead, which has been designated Lead IV. In principle, one electrode, known as "the exploring electrode," is placed on the front of the chest over the precordia, and the other, known as "the indifferent electrode," is placed either upon one limb (usually the left leg), or on the back below the scapula. The exploring electrode is standardised so that a negative change of potential is recorded as a downward stroke. There are many varieties of Lead IV, dependent upon the actual position in which these two electrodes are placed, but it will not be necessary to discuss these here. They may be found by reference to standard electrocardiographic works. The essential difference between the chest lead and the standard lead is that the exploring electrode is placed in a very much closer relationship to the heart, and thus changes in electrical potential are more easily discoverable.

There are certain characteristics of the normal chest lead which must be noted, before judging the possible abnormalities. Firstly, the $\mathbf{P}$ wave may be either upward, bifid, or inverted; the $P$ wave is particularly clear-cut when the chest lead is placed at the right border of the sternum at the third intercostal space. The PR interval is often shorter than that found in the normal standard leads taken from the same case. Secondly, the $R$ wave is always present, and is of a height greater than 2 millimetres. This wave is smallest at the right border of the heart, becoming progressively larger as the exploring electrode is moved towards the apex. Thirdly, the S wave shows the opposite behaviour. Fourthly, the ST interval is usually very short and does not vary from the isoelectric line for more than 2 millimetres in an upward direction and not at all in a downward direction. Fifthly, the $T$ wave is universally upright, being tallest at the apex and decreasing as the exploring electrode is moved towards the right border of the heart. There is one exception, however, to this rule; a chest lead taken from a child of seven years of age, or under, always shows an inverted $T$ in any lead taken from over the precordial area. This is of no pathological significance.

A record which does not conform to the above pattern is pathological. The usual pathological findings are either an absent $R$ wave; or an $R$ wave of less than 2 millimetres in height; a deviation of the ST segment in an upward or downward direction, or a take off of the $T$ wave 
from any point greater than 2 millimetres above the isoelectric line; or any deviation of this take off in a downward direction; lastly, an inverted $T$ wave in an adult is always of pathological significance.

In considering diseases of the coronary arteries, it must be remembered that a great variety of functional disorders may result from this disease process, and therefore the finding of a degree of heart block, or auricular fibrillation, may be the only evidence of coronary disease. An acute occlusion of the coronary arteries, that is, acute coronary thrombosis, does, however, produce certain changes in the QRST complex which are diagnostic of this condition. Firstly, there is an absence of the normal isoelectric period between $\mathrm{S}$ and $\mathrm{T}$ waves, producing, therefore, the "plateau" type of curve in which the ST segment is either elevated or depressed (Fig. 5.)

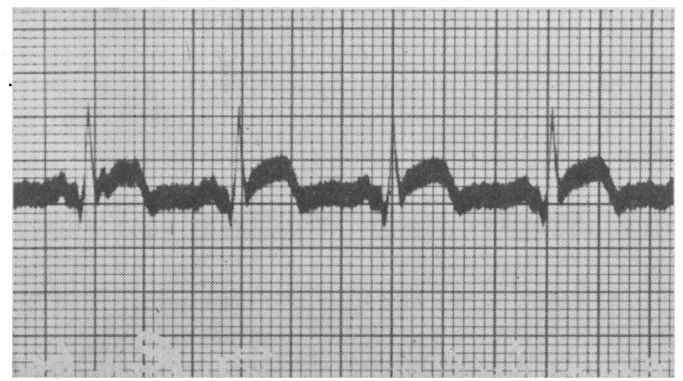

Frg. 5.-Acute coronary thrombosis. Lead IV. Note take off of the $T$ wave from above isoelectric line and its "plateau" shape.

This is known as the ST deviation, and two varieties occur: (I) T takes off from the R or S waves from a plateau shaped elevation in Lead I and from a plateau shaped depression in Lead III, known as the $T_{1}$ type of coronary thrombosis, and (2) the take off of the plateau shaped depression occurs in Lead I and the upward elevation occurs in Lead III, known as the $T_{3}$ type of coronary thrombosis. Either of these are the typical findings on the electrocardiogram when taken from a case of acute coronary thrombosis which has occurred during the previous day or two. About a week later, an electrocardiogram taken from the same patient shows that the $T$ wave is returning towards the isoelectric line, and is increasing in voltage (Fig. 6). As this process goes on, the $\mathrm{T}$ wave deflects in the opposite direction to the original $\mathrm{RT}$ deviation in the respective leads. These typical curves may, however, be absent, and no change may be found except •

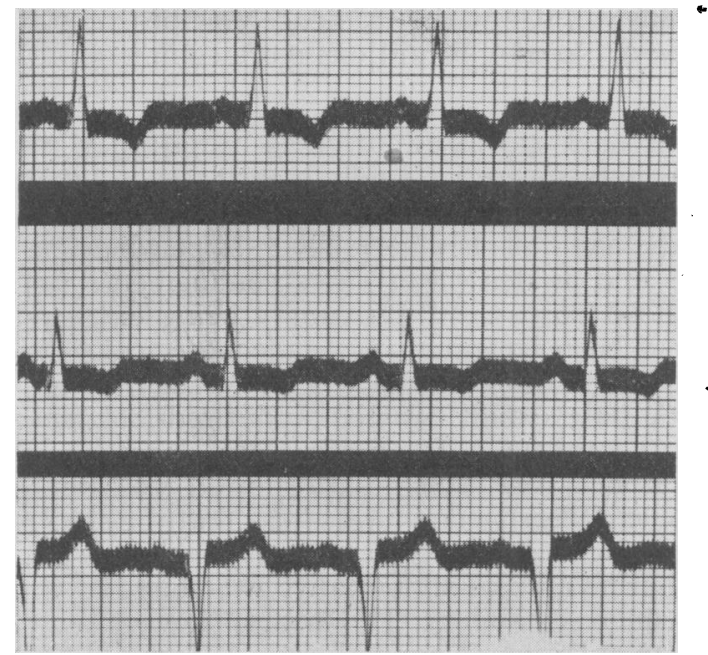

Frg. 6.-Acute coronary thrombosis $T_{1}$ type-a week later. Note inversion of $T_{1}$ and $T_{2}$ with upright $T_{3}$. The RT segment is slightly depressed from isoelectric line in Leads I and II, and slightly elevated in Lead I.II. 
by the exhibition of Lead IV. In this case, the electrocardiographic tracing will show some abnormality, as described above.

There are, however, other changes which may occur in the electrocardiogram when the ventricular muscle has been affected by coronary changes. The following are some of the more typical changes, any, or groups of which, may of course be present in one particular tracing.

I. There may be a notching or slurring of the QRS complex.

II. There may be a prolonged duration of the QRS complex, i.e. it may be spread out over a period longer than $0 \cdot I$ second.

III. The voltage of the QRS may be less than 0.5 millivolt.

IV. The $T$ wave may be inverted, that is, it may turn downwards in Leads I or II, or both. An inverted $T$ wave in Lead III usually has no pathological importance unless accompanied by inversion in I or II of the other leads. There is one exception to this rule, however, and that is in the later stages of a $T_{3}$ type of coronary thrombosis. In this type of coronary thrombosis there will be a large inverted $T$ in Lead III, with a large, upright $T$ in Lead $I$. The differentiation is made upon the history, upon previous electrocardiographic findings, and by noting the increased amplitude of these $T$ waves.

V. Another change that may occur in the $T$ wave is that the voltage may be less than $I$ millivolt.

VI. The ST interval may arise from the proper level, but the graph may pass to the peak of the $T$ wave by an abnormal course, leading to a deformity of the $T$ wave. line.

VII. The ST segment may be abnormally elevated above, or depressed below the isoelectric

These, then, are some of the changes which may be present on the electrocardiogram taken from a heart which is the seat of coronary disease.

We have made no attempt to deal with the subject of electrocardiography in its entirety, but we have tried to point the way.

\section{Clinical Page}

By STELLA INSTONE, M.D., M.R.C.P. Medical Registrar, Elizabeth Garrett Anderson Hospital

\section{Case 1.-A CASE OF ENDOCARDITIS LENTA WITH INCONSPICUOUS SYMPTOMS}

The following case illustrates how the existence and progress of a subacute infective endocarditis may sometimes be masked by conditions more common but less serious.

\section{CASE NOTE}

History: A housewife aged 37 was admitted to hospital for investigation in August, I942. She complained of increasingly severe lassitude, breathlessness upon exertion, insomnia, giddiness, night-sweats and amenorrhoea for the past year, with loss of appetite and of weight. Twelve years previously she had had rheumatic fever, since when she had been liable to frequent rheumatism in the wrists and ankles. Her three confinements were uneventful, the last one being in I936. The financial position of her family being extremely poor, the patient's diet for the past few years had been irregular and inadequate, consisting largely in milk, tea and fruit, with very little bread and potatoes, and no meat, bacon, cheese or vegetables.

Clinical findings: A pale, small, wasted and tired-looking woman with flushed face, toxic skin, and a dry furred tongue. Temperature 99 degrees, pulse roo. Respiration rate 20 , B.P. IOo/6o, weight 5 stone 4 lbs. The mouth showed gross dental sepsis with spongy gums, which bled on examination and were incompletely healed at the sites of recent dental extractions. The buccal mucous membrane showed scattered patches of thrush. The chest wall showed signs of previous rickets, but no clinical abnormality was found in the respiratory system, apart from slight but definite finger-clubbing. The heart showed an established mitral stenosis with no evidence of cardiac enlargement or failure. Abdominal examination revealed marked tenderness in both renal angles, but no other abnormality. Pelvic examination was negative as was that of the central nervous system. 